\title{
Heat conduction and phonon localization in disordered harmonic crystals
}

\author{
Anupam Kundu,${ }^{1}$ Abhishek Chaudhuri,${ }^{2}$ Dibyendu Roy,${ }^{3}$ Abhishek Dhar,${ }^{1}$ Joel L. Lebowitz ${ }^{4}$ and Her- \\ BERT SPOHN ${ }^{5}$ \\ 1 Raman Research Institute - C.V. Raman Avenue, Bangalore 560080, India \\ 2 Department of Physics and Astronomy - University of Sheffield, Sheffield S3 7RH, United Kingdom \\ 3 Department of Physics - University of California-San Diego, La Jolla, CA 92093 \\ 4 Departments of Mathematics and Physics - Rutgers University, Piscataway, NJ 08854 \\ 5 Zentrum Mathematik - Technische Universität München, D-85747 Garching, Germany \\ PACS nn.mm.xx - 05.60.Cd,44.10.+i,63.50.-x
}

\begin{abstract}
We investigate the steady state heat current in two and three dimensional isotopically disordered harmonic lattices. Using localization theory as well as kinetic theory we estimate the system size dependence of the current. These estimates are compared with numerical results obtained using an exact formula for the current given in terms of a phonon transmission function, as well as by direct nonequilibrium simulations. We find that heat conduction by high-frequency modes is suppressed by localization while low-frequency modes are strongly affected by boundary conditions. Our heuristic arguments show that Fourier's law is valid in a three dimensional disordered solid except for special boundary conditions. We also study the pinned case relevant to localization in quantum systems and often used as a model system to study the validity of Fourier's law. Here we provide the first numerical verification of Fourier's law in three dimensions. In the two dimensional pinned case we find that localization of phonon modes leads to a heat insulator.
\end{abstract}

Transport in random media is a topic of great current interest 1 -7]. Here we study the effect of isotopic mass disorder and boundary conditions on heat conduction and localization in two $(2 D)$ and three $(3 D)$ dimensional harmonic crystals, where the properties of the ordered system are known exactly. These are systems of $N^{d}(d=2,3)$ atoms in contact, at their surfaces perpendicular to the $x$-axis, with heat reservoirs at different temperatures. We shall focus mainly on the dependence of the heat flux $J$ on $N$. When Fourier's law holds then $J \sim N^{-1}$ but this is known to be violated in computer simulations 8 and some experiments 9,10 in $1 D$ and $2 D$ systems where one finds $J \sim N^{-\mu}$ with $\mu \neq 1$. Earlier work $[7,11]$ on heat conduction in glassy harmonic systems computed the frequency-dependent thermal diffusivity using the GreenKubo formula for different system sizes. However their study did not directly address the question of asymptotic size dependence of conductivity.

We will first describe the precise model studied, then give our heuristic arguments for calculating the $N$ dependence of $J$ and finally present the numerical results.

We consider simple cubic lattices with displacements at each lattice site $\mathbf{n}\left(n_{\nu}=1,2, \ldots, N\right.$ for $\left.\nu=1,2, \ldots, d\right)$ given by a scalar variable $x_{\mathbf{n}}$. In the harmonic approximation the system Hamiltonian is given by

$$
\begin{aligned}
H & =\sum_{\mathbf{n}} \frac{m_{\mathbf{n}}}{2} \dot{x}_{\mathbf{n}}^{2}+\sum_{n_{1}=1}^{N-1} \sum_{\mathbf{n}^{\prime}, \hat{\mathbf{e}}} \frac{k}{2}\left(x_{\mathbf{n}}-x_{\mathbf{n}+\hat{\mathbf{e}}}\right)^{2} \\
& +\sum_{\mathbf{n}^{\prime}} \frac{k^{\prime}}{2}\left(x_{1, \mathbf{n}^{\prime}}^{2}+x_{N, \mathbf{n}^{\prime}}^{2}\right)+\sum_{\mathbf{n}} \frac{k_{o}}{2} x_{\mathbf{n}}^{2}
\end{aligned}
$$

where $\hat{\mathbf{e}}$ refers to the $d$ unit vectors and we have denoted $\mathbf{n}=\left(n_{1}, \mathbf{n}^{\prime}\right)$. We impose periodic boundary conditions (BCs) in the directions $\nu=2,3, \ldots, d$ and consider two different $\mathrm{BCs}$ in the direction of heat conduction $(\nu=1)$ : (i) fixed BCs $k^{\prime}>0$ and (ii) free BCs $k^{\prime}=0$. Pinning refers to the case with $k_{o}>0$. We consider binary mass disorder with equal number of particles of masses $\bar{m}-\Delta$ and $\bar{m}+\Delta$ distributed randomly on the lattice sites. The equations of motion of particles in the bulk $\left(1<n_{1}<N\right.$ ) are given by: $m_{\mathbf{n}} \ddot{x}_{\mathbf{n}}=-\sum_{ \pm \hat{\mathbf{e}}} k\left(x_{\mathbf{n}}-x_{\mathbf{n}+\hat{\mathbf{e}}}\right)-k_{o} x_{\mathbf{n}}$. The particles at the surfaces $n_{1}=1$ and $n_{1}=N$ are connected to heat reservoirs, at temperatures $T_{L}$ and $T_{R}$ respectively. These are modeled by white noise Langevin 
equations. This means that particles at $n_{1}=1$ and $n_{1}=$ $N$, have additional forces given respectively by $\left(-k^{\prime} x_{\mathbf{n}}-\right.$ $\left.\gamma \dot{x}_{\mathbf{n}}+\eta_{\mathbf{n}}^{L}\right)$ and $\left(-k^{\prime} x_{\mathbf{n}}-\gamma \dot{x}_{\mathbf{n}}+\eta_{\mathbf{n}}^{R}\right)$, where $\eta^{L}$ and $\eta^{R}$ are noise terms with strengths proportional to $T_{L}$ and $T_{R}$ respectively and to the friction constant $\gamma$. If we model the heat reservoirs themselves by infinite ordered harmonic crystals then Langevin type equations for the system 12 are obtained on eliminating the bath degrees of freedom. The two different BCs then emerge naturally. Fixed BCs correspond to reservoirs with properties different from the system (e.g. different spring constants) while free BCs correspond to the case where the reservoir is simply an extension of the system (without disorder) 6, 13, 14. We note that fixed BCs are more realistic.

In the nonequilibrium steady state, the heat current per unit area (assuming unit lattice spacing length) from the left to the right reservoir is given by $[12,15$ :

$$
\mathcal{J}=\frac{\Delta T}{2 \pi N^{d-1}} \int_{0}^{\infty} d \omega \mathcal{T}(\omega)
$$

where $\Delta T=T_{L}-T_{R}$ and $\mathcal{T}(\omega)$ is the transmission coefficient of phonons at frequency $\omega$ from the left to the right reservoir. Our interest here are in the disorder averaged transmission $T(\omega)=[\mathcal{T}(\omega)] / N^{d-1}$ and the current $J=[\mathcal{J}]$.

The phonon and electron localization problems are closely related. For the system without baths consider the displacement field $a_{\mathbf{n}}(p)$ for the $p^{\text {th }}$ normal mode. This satisfies the equation: $m_{\mathbf{n}} \omega_{p}^{2} a_{\mathbf{n}}=\left(2 d+k_{o}\right) a_{\mathbf{n}}-\sum_{\hat{\mathbf{e}}} a_{\mathbf{n}+\hat{\mathbf{e}}}$. By introducing variables $\psi_{\mathbf{n}}(p)=m_{\mathbf{n}}^{1 / 2} a_{\mathbf{n}}(p), v_{\mathbf{n}}=(2 d+$ $\left.k_{o}\right) / m_{\mathbf{n}}$ and $t_{\mathbf{n}, 1}=1 /\left(m_{\mathbf{n}} m_{\mathbf{l}}\right)^{1 / 2}$ for nearest neighbour sites $\mathbf{n}, \mathbf{l}$ this equation transforms to a Schrodinger-type equation $\omega_{p}^{2} \psi_{\mathbf{n}}(p)=v_{\mathbf{n}} \psi_{\mathbf{n}}(p)-\sum_{\mathbf{l}} t_{\mathbf{n}, 1} \psi_{\mathbf{l}}(p)$. The nearest neighbour hopping $t_{\mathbf{n}, \mathbf{l}}$ and on-site energies $v_{\mathbf{n}}$ are now correlated random variables. For the unpinned case with $k_{o}=0$, translation invariance gives rise to extended modes at low frequencies. The pinned case has no translational invariance and is closer to the usual electron localization problem.

The effect of disorder on heat conduction in a harmonic crystal manifests itself in two ways: (i) Anderson localization 16 of phonon modes of frequency $\omega$ will make them non-conducting, (ii) non-localized phonons will be scattered by the impurities. From Eq. (2) the net current is given by the integrated transmission and for large system sizes the integral is over the range of normal modes. It is natural to classify normal modes as localized, diffusive or ballistic. For modes localized on a length scale $\ell$, $T(\omega) \sim e^{-N / \ell}$. This $\ell$ depends on the phonon frequency and low frequency modes for which $\ell(\omega) \gtrsim N$ will therefore be carriers of the heat current. Diffusive modes are spatially extended but non-periodic and $T(\omega) \sim 1 / N$. Ballistic modes are extended and approximately periodic and their transmission is $N$-independent.

A renormalization group study in a disordered continuum elastic model by John etal 17] found that in 1D and
2D all non-zero frequency phonons are localized. They studied the spreading of an energy pulse to define a frequency dependent diffusivity $D_{0}(\omega)$. From the behaviour of $D_{0}(\omega)$ under renormalization one can obtain a differential recursion relation for the resistivity. This relation shows that in the large system size limit the RG flow is towards infinity for dimensions $\leq 2$ in contrast to $d=3$ where the flow is towards zero as long as $\omega$ is less than some fixed value, independent of system size. Hence all finite-frequency modes in one and two dimensions are localized. From the differential recursion relations one finds that for $d=1$ and $d=2$ the localization length in $\omega \rightarrow 0$ limit diverges as $\sim 1 / \omega^{2}$ and $\sim e^{1 / \omega^{2}}$ respectively. In $3 D$ there exists a frequency, independent of system size, above which all states are localized while states below that frequency are extended. Hence for a system of size $N$ there will be a cut-off frequency $\omega_{c}^{L}$ (which depends on $N$ for $d \leq 2$ ) above which all the modes are localized. In different dimensions $\omega_{c}^{L}$ is given by

$$
\begin{aligned}
\omega_{c}^{L} & \sim N^{-1 / 2} \text { for } d=1 \\
& \sim[\log (N)]^{-1 / 2} \text { for } d=2 \\
& \sim \text { nonzero value independent of } N \text { for } d=3
\end{aligned}
$$

For extended modes with $\omega<\omega_{c}^{L}$ we use kinetic theory to determine the cut-off frequency $\omega_{c}^{K}$ below which states are ballistic. Rayleigh scattering of phonons gives a mean free path $\ell_{K}(\omega) \sim \omega^{-(d+1)}$ in $d$-dimensions. This gives $\omega_{c}^{K}=N^{-1 /(d+1)}$ below which $\ell_{K}(\omega) \gtrsim N$.

The net current in the system consists of both ballistic $\left[J_{B} \sim \int_{0}^{\omega_{c}^{K}} d \omega T(\omega)\right]$ and diffusive $\left[J_{D} \sim \int_{\omega_{c}^{K}}^{\omega_{c}^{L}} d \omega T(\omega)\right)$ contributions, $J=J_{B}+J_{D}$. One other crucial observation, consistent with the numerics, is that $T(\omega)$ in the ballistic region has the same form as for an ordered system, which is sensitive to boundary conditions.

In $1 D$ both $\omega_{c}^{K}, \omega_{c}^{L} \sim N^{-1 / 2}$ and numerical studies show that all extended states are ballistic 18. For free BC $T(\omega) \rightarrow$ const. as $\omega \rightarrow 0$ and hence $J \sim \int_{0}^{N^{-1 / 2}} d \omega \sim$ $N^{-1 / 2}$. For fixed BC $T(\omega) \sim \omega^{2}$ leading to $J \sim N^{-3 / 2}$. The dependence of the form of $T(\omega)$ on pinning was discussed in 19 in detail. Thus surprisingly one finds a strong dependence on BCs. In the presence of pinning, the low frequency modes are removed and $J \sim e^{-c N}$. These results agree with earlier rigorous and numerical work on this system $13,15,18,20$. We now calculate the asymptotic system size dependence in $2 D$ and $3 D$.

Unpinned lattice: To find the ballistic contribution we note that for the ordered lattice $T(\omega) \sim \omega^{d-1}$ as $\omega \rightarrow 0$ for free BCs and as $T(\omega) \sim \omega^{d+1}$ for fixed BC. This can be seen in the following way. We write $\mathbf{n}=\left(n_{1}, \mathbf{n}^{\prime}\right)$ and $\mathbf{q}=$ $\left(q_{2}, q_{3}, \ldots, q_{d}\right)$ with $q_{\alpha}=\frac{2 \pi n}{N}$ where $n$ goes from 1 to $N$. Now if we define $x_{n_{1}}(\mathbf{q})=N^{-(d-1) / 2} \sum_{\mathbf{n}^{\prime}} x_{\left(n_{1}, \mathbf{n}^{\prime}\right)} e^{i \mathbf{q} \cdot \mathbf{n}^{\prime}}$, then one can show that, for each $\mathbf{q}, x_{l_{1}}(\mathbf{q})$ satisfies a Langevin equation corresponding to a one-dimensional Hamiltonian with the onsite spring constant $k_{o}$ replaced by $\lambda(\mathbf{q})=k_{o}+2 k\left(d-1-\sum_{\alpha=2, d} \cos \left(q_{\alpha}\right)\right)$. This means 
Heat conduction and phonon localization in disordered harmonic crystals
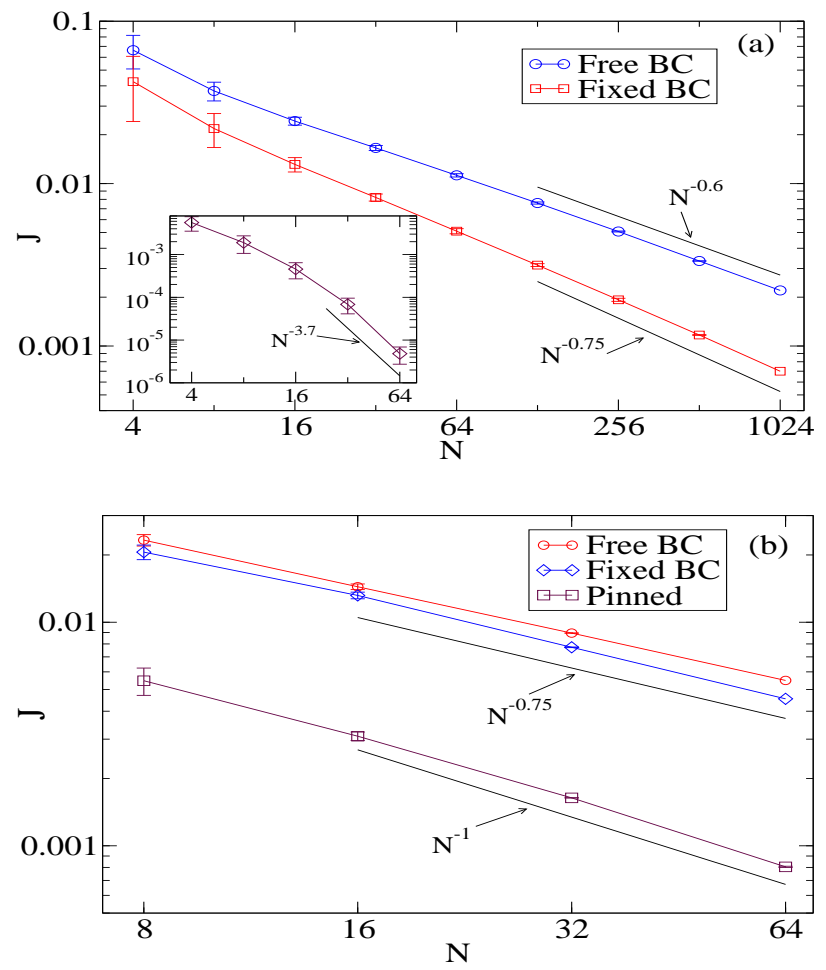

Fig. 1: Plot of the $N$-dependence of disorder averaged current $J$. (a) $2 D$ : Inset shows result for pinned case. For free BC $\Delta=0.8$ and for fixed BC $\Delta=0.95$. For pinned case $\Delta=$ $0.4, k_{o}=10.0$. (b) $3 D$ : For all cases $\Delta=0.8$ and for pinned case $k_{o}=10.0$. Error bars show standard deviations due to sample-to-sample fluctuations and are very small except in the $2 D$ pinned case.

that the problem of heat conduction in a $d$-dimensional ordered harmonic lattice can be related to heat conduction across $N^{d-1}$ independent ordered harmonic chains with different onsite potentials 21,23 . Hence the transmission coefficient $T(\omega)$ for the $d$-dimensional lattice can be expressed as a sum of the transmission coefficients of the $1 D$ ordered chains. Using this result and the analytic form of the transmission coefficient for the $1 D$ chain 23 we find in the $N \rightarrow \infty$ and $\omega \rightarrow 0$ limit

$$
\begin{aligned}
T(\omega) & \sim \omega^{d-1} \text { for open boundary condition } \\
& \sim \omega^{d+1} \text { for fixed boundary condition. }
\end{aligned}
$$

Hence we get the ballistic contribution to the total current density (for the unpinned case) as: $J_{B} \sim \int_{0}^{\omega_{c}^{K}} d \omega \omega^{d-1} \sim$ $N^{-d /(d+1)}$ for free BC and $J_{B} \sim \int_{0}^{\omega_{c}^{K}} d \omega \omega^{d+1} \sim$ $N^{-(d+2) /(d+1)}$ for fixed BC. In $2 D$ using kinetic theory and localization theory we expect localized modes for $\omega \gtrsim$ $\omega_{c}^{L}=(\ln N)^{-1 / 2}$, ballistic modes for $\omega \lesssim \omega_{c}^{K}=N^{-1 / 3}$ and diffusive modes for $\omega_{c}^{K} \lesssim \omega \lesssim \omega_{c}^{L}$. The diffusive contribution to total current will scale as $J_{D} \sim(\ln N)^{-1 / 2} N^{-1}$. As argued above, the ballistic contribution depends on BCs with $J_{B} \sim N^{-4 / 3}$ for fixed BC and $J_{B} \sim N^{-2 / 3}$ for free BC. Hence, adding all the contributions, we conclude

\begin{tabular}{|c|c|c|c|c|}
\hline & \multicolumn{2}{|c|}{$d=2$} & \multicolumn{2}{c|}{$d=3$} \\
\cline { 2 - 5 } & Heuristic & Numerical & Heuristic & Numerical \\
\hline Pinned & $\exp (-b N)$ & $N^{-3.7}$ & $N^{-1}$ & $N^{-1.0}$ \\
\hline Fixed & $N^{-1}(\ln N)^{-1 / 2}$ & $N^{-0.75}$ & $N^{-1}$ & $N^{-0.75}$ \\
\hline Free & $N^{-2 / 3}$ & $N^{-0.6}$ & $N^{-3 / 4}$ & $N^{-0.71}$ \\
\hline
\end{tabular}

Table 1: The table gives the $N$ dependence of $J$ and summarizes the main results of the paper. The error bar for the numerically obtained exponent values is of the order \pm 0.02 . N.B: The system sizes used may be far from asymptotic.

that asymptotically: $J \sim(\ln N)^{-1 / 2} N^{-1}$ for fixed $\mathrm{BC}$ and $J \sim N^{-2 / 3}$ for free BC. In $3 D$ we expect that $\omega_{c}^{L}$ is independent of $N$ and states with $\omega>\omega_{c}^{L}$ are localized. Extended modes with $\omega \lesssim \omega_{c}^{K}=N^{-1 / 4}$ are ballistic while those with $\omega_{c}^{K} \lesssim \omega \lesssim \omega_{c}^{L}$ are diffusive. The contribution from the diffusive modes scales as $J_{D} \sim N^{-1}$ while the ballistic contribution again depends on boundary conditions with $J_{B} \sim N^{-5 / 4}$ for fixed BC and $J_{B} \sim N^{-3 / 4}$ for free BC. We conclude that asymptotically: $J \sim N^{-1}$ for fixed $\mathrm{BC}$ and $J \sim N^{-3 / 4}$ for free BC.

Pinned lattice: There is now a gap in the spectrum, starting from $\omega=0$, and thus there are no low frequency ballistic modes. In $2 D$ localization theory then tells us that for sufficiently large $N$, all non-zero frequency modes become localized, hence we should get an insulator. In $3 D$ there is a finite band of diffusive states and therefore we expect a normal conductor satisfying Fourier's law.

Numerical results: We now check the above predictions through extensive numerical calculations. We measure force-constants in units of $k$, masses in units of the average mass $\bar{m}$, time in units of the inverse frequency $\Omega^{-1}=(\bar{m} / k)^{1 / 2}$, displacements in units of the lattice spacing $a$, friction constant $\gamma$ in units of $\bar{m} \Omega$, and temperature in units of $\bar{m} a^{2} \Omega^{2} / k_{B}$. We set $k=1, \bar{m}=1$ and we fixed $\Delta T=1, \gamma=1$. For fixed BCs and pinned cases $k^{\prime}=1$. Different values of the mass variance $\Delta$ and the on-site spring constant $k_{o}$ were studied for $2 D$ and $3 D$ lattices of different sizes 24. Here we report results for cases with the strongest disorder. The transmission coefficient $\mathcal{T}_{N}(\omega)$ can be expressed in terms of phonon Green's functions and can be accurately determined numerically using a transfer matrix representation 24. By performing a discrete sum over the transmitting range of frequencies we evaluate the integration in Eq. (2) to obtain $\mathcal{J}$. For $N \geq 64$ in $3 D$ the transfer matrix method has numerical problems and in those cases we performed nonequilibrium simulations to find the heat current.

We also studied the properties of the normal modes of the isolated harmonic lattices with fixed BCs. We measure the degree of localization of a given mode by the inverse participation ratio defined as $P^{-1}=\sum_{\mathbf{n}} a_{\mathbf{n}}^{4} /\left(\sum_{\mathbf{n}} a_{\mathbf{n}}^{2}\right)^{2}$. For an extended state $P^{-1}$ is of order $\mathcal{O}\left(N^{-d}\right)$ while for a localized state, it is $\mathcal{O}(1)$.

In Fig. (1) we show the $N$-dependence of the disorder averaged current $J=[\mathcal{J}]$ in $d=2,3$. Our findings and the comparison with the heuristic predictions are summarized 

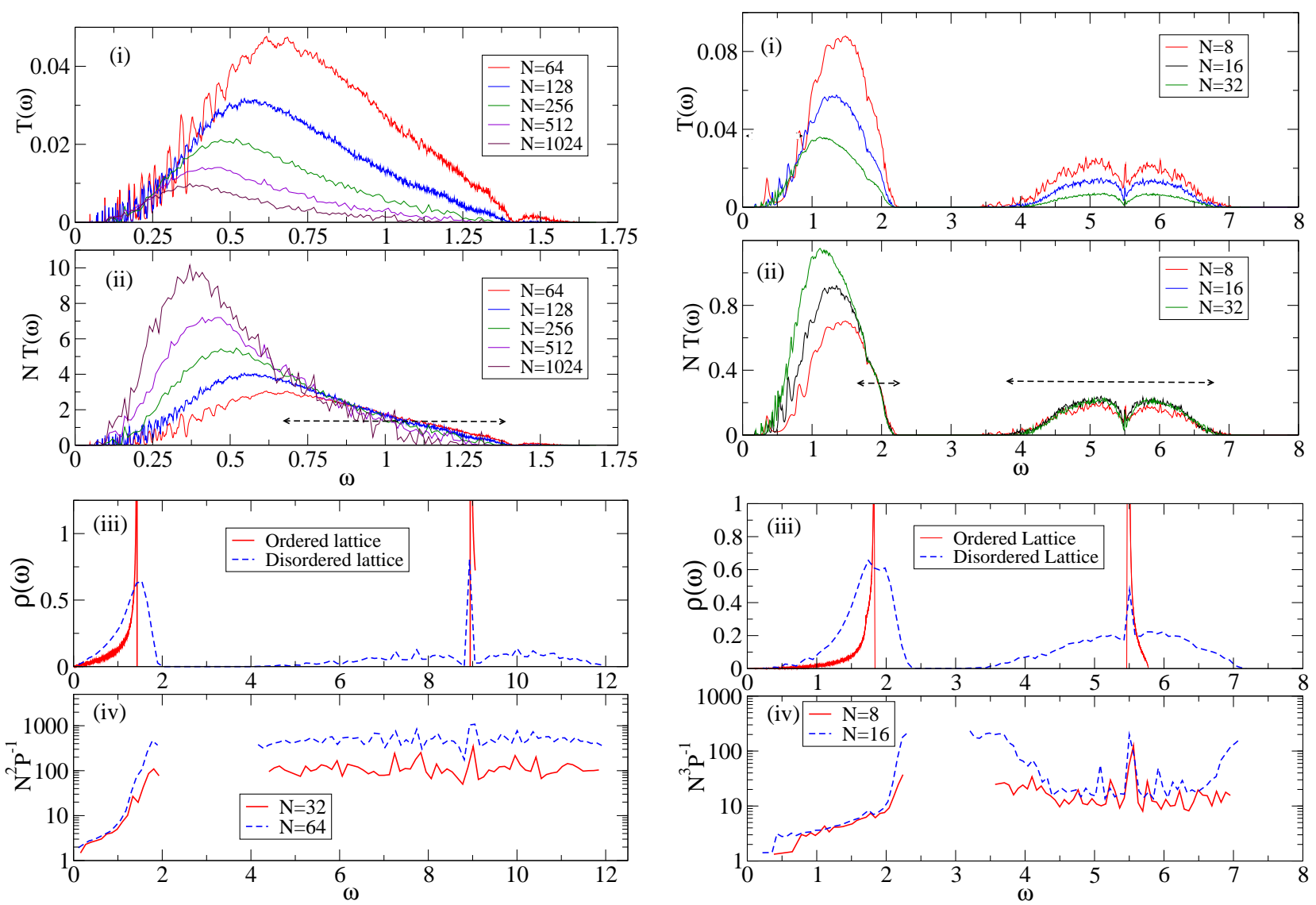

(a) Unpinned $2 D$ lattice

(b) Unpinned $3 D$ lattice

Fig. 2: (i) Plot of $T(\omega)$. (ii) Plot of $N T(\omega)$. The range of frequencies for which $T(\omega) \sim 1 / N$ is indicated by arrowed lines and corresponds to diffusive modes. (iii) Plot of $\rho(\omega)$ for binary mass ordered lattice and single disordered sample. (iv) Plot of $N^{d-1} \times P^{-1}$ for single samples. Non-collapse of plots for different $N$ in (iv) indicate localized states.

in Table (1). The results for the free $\mathrm{BC}$ case are consistent with the heuristic predictions while those for fixed BC show significant deviations. For the pinned system we obtain, in agreement with the theoretical prediction. In $2 \mathrm{D}$ we find a strong decay of the current with $N$ with $\mu=3.7$ suggesting that at larger system sizes we will get an exponential decay corresponding to a heat insulator. In $3 \mathrm{D}$ we find $\mu=1$ implying that the system is a normal heat conductor. For the binary mass distribution we do not find a transition to insulating behaviour with increasing disorder.

The $N$-dependence of the disorder-averaged phonon transmission coefficient $T(\omega)=\left[\mathcal{T}_{N}(\omega)\right] / N^{d-1}$ sheds additional light on the nature of phonons in different frequency regions. For the case of fixed $\mathrm{BCs}$ we show in Fig. (2) results for transmission, density of states and inverse participation ratios and from these we can see the range of allowed modes and their degree of localization. By plotting $N T(\omega)$ we identify the diffusive regime. From plots (iii-iv) we see that in both $2 D$ and $3 D$ there are effectively two phonon bands, a remnant of the ordered binary mass case. In $2 D$ the upper band is fully localized while in $3 D$ there is a small number of localized states near the band edges. In $2 D$ the lower band has extended states below a cut-off $\omega_{c}^{L}$ which decreases slowly with $N$. In both $2 D$ and $3 D$ the lower band has diffusive and ballistic states and the crossover scale $\omega_{c}^{K}$ decreases with system size. The expected $N$-dependence of $\omega_{c}^{K}$ and $\omega_{c}^{L}$ are difficult to verify at these system sizes.

For the case of free BCs, we find that the values of $T(\omega)$ in the diffusive regime matches with those for fixed BCs but are completely different in the ballistic regime. This is seen in Fig. (3) where we plot the effective mean free path $l_{\text {eff }}(\omega)=N T(\omega) / \omega^{d-1}$ in the low-frequency region. The difference between free and fixed BCs is larger in $2 D$ than in $3 D$ and explains the similar feature observed for the $J$ values in Fig. (1). For free BC, $l_{\text {eff }}$ is roughly consistent with the kinetic theory prediction $l_{\text {eff }}^{-1} \sim N^{-1}+\ell_{K}^{-1}(\omega)$ but the behaviour for fixed $\mathrm{BC}$ is very different. The inset of Fig. (3) plots $l_{\text {eff }}$ for the equal mass ordered case and we find that in the ballistic regime it is very close to the disordered case, an input that we used in the heuristic derivation. The oscillations in the transmission for fixed $\mathrm{BC}$ arise from scattering and interference of waves at the 


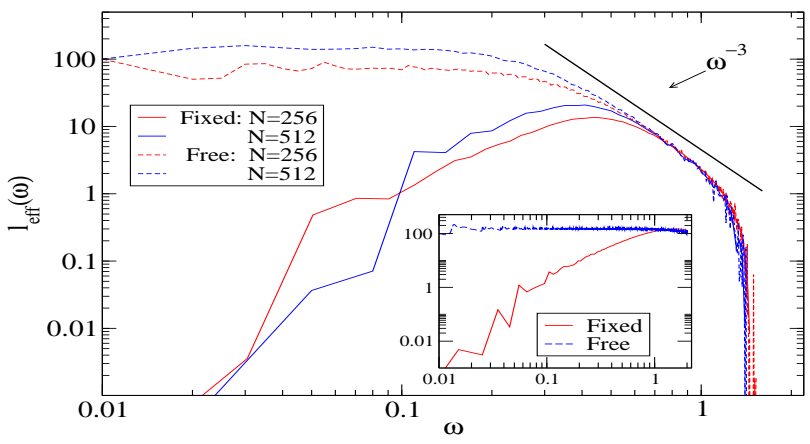

(a) $2 D$ lattice

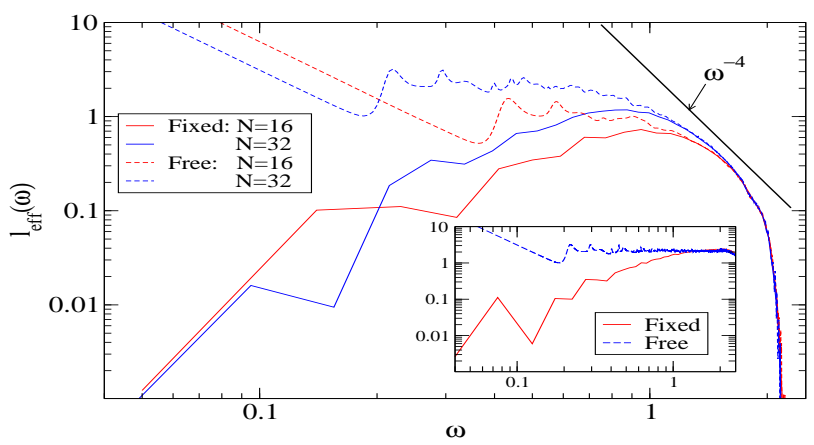

(b) $3 D$ lattice

Fig. 3: Plot of the effective mean-free path $l_{\text {eff }}=N T(\omega) / \omega^{d-1}$ in (a) $2 D$ and (b) $3 D$ with $\Delta=0.8$. The insets show $\ell_{\text {eff }}$ for the ordered lattices with a single mass. An $\omega^{-(d+1)}$ behaviour is observed in a small part of the diffusive region. The fixed BC data is highly oscillatory and has been smoothed.

interfaces. For the fully pinned case we find $T(\omega) \sim e^{-b N}$ in $2 D$ and as $T(\omega) \sim 1 / N$ in $3 D$ 24].

In summary the analytic arguments show that the contribution of ballistic modes to conduction is dependent on $\mathrm{BCs}$ and is strongly suppressed for fixed BCs, the more realistic case. In $3 D$ this leads to diffusive modes dominating for large system sizes and Fourier's law is satisfied. Thus a finite heat conductivity may be obtained without invoking anharmonicity as is usually believed to be necessary [25]. Our numerical results verify the predictions for free $\mathrm{BCs}$ and we believe that much larger system sizes are necessary to verify the fixed $\mathrm{BC}$ results (this is also the case in $1 D[18]$ ). It is also possible that the assumptions made to predict the asymptotic $N$ dependence of current is not correct. We are uncertain at present whether the disagreements are due to finite size effects or to the inadequacy of the theory. A number of recent experiments have directly observed localization of electrons [4], photons 2], matter waves [5] and acoustic waves [3]. Our study shows clearly that the ballistic and diffusive contributions to the heat current would make it difficult to observe localization effects in heat conduction studies 6, 14]. Our study of the pinned system gives us a clearer understanding of the role of low frequency modes in giving rise to diverging thermal conductivity and provides the simplest example of a
$3 D$ deterministic system with diffusive transport. The $2 D$ pinned system is a heat insulator and thin films attached on insulating substrates may show such behaviour.

We thank G. Baskaran, M. Aizenman, T. Spencer and especially D. Huse for useful discussions. We also thank S. Sastry and V. Vasisht for use of computational facilities. The research of J. L. Lebowitz was supported by NSF grant No. DMR0802120 and by AFOSR grant No. FA9550-07.

\section{REFERENCES}

[1] Chang C. W. et al, Phys. Rev. Lett., 97, (2006), 085901.

[2] Schwartz T. et al, Nature, 446,(2007), 52-55.

[3] Hu H. et al, Nature Phys., 4, (2008), 945-948.

[4] Gómez-Navarro C. et al, Nature Materials, 4,(2005), 534 -539 .

[5] Billy J. et al, Nature, 453, (2008), 891-894.

[6] Savic I. and Mingo N. and Stewart D. A., Phys. Rev. Lett., 101,(2008), 165502.

[7] Xu N. et al, ibid., 102,(2009), 038001.

[8] Dhar A., Adv. Phys., 57,(2008), 457.

[9] Chang C. W. et al, Phys. Rev. Lett., 101,(2008), 075903.

[10] Nika D.L. et al, Appl. Phys. Lett., 94,(2009), 203103.

[11] Allen P. B. and Feldman J.L., Phys. Rev. Lett., 62,(1989), 645. Feldman J. L. , Allen P. B., and Bickham S. R., Phys. Rev. B, 59, (1999), 3551. Feldman J. L., Kulge M. D., Allen P. B. and Wooten F., Phys. Rev. B, 48, (1993), 12589.

[12] Dhar A. and Roy D., J. Stat. Phys., 125,(2006), 805.

[13] Rubin R. J. and Greer W. L., J. Math. Phys., 12,(1971), 1686.

[14] Stoltz G., Lazzeri M. and Mauri F., Jn. Phys. Cond. Matt., 21,(2009), 245302.

[15] Casher A. and Lebowitz J. L., J. Math. Phys., 12,(1971), 1701.

[16] Anderson P. W., Phys. Rev., 109, (1958), 1492-1505.

[17] John S., Sompolinsky H., and Stephen M. J., Phys. Rev. B, 27, (1983), 5592.

[18] Dhar A., Phys. Rev. Lett., 86, (2001), 5882.

[19] Roy D. and Dhar A., Phys. Rev. E, 78, (2008), 051112.

[20] Matsuda H. and Ishin K., Prog. Theor. Phys. Suppl., 45, (1970), 56 .

[21] Nakazawa H., Progress of Theoretical Physics, 39, (1968), 239.

[22] Nakazawa H., Progress of Theoretical Physics Supplement, 45, (1970), 231.

[23] Roy D. and Dhar A., J. Stat. Phys., 131, (2008), 535.

[24] Chaudhuri A. et al, arXiv:0902.3350.,

[25] Ziman J. M., Principles of the Theory of Solids (Cambridge University Press, Cambridge) 1972. 